\title{
Las Pailas. Nuevos aportes para la comprensión de la agricultura prehispánica en el Valle Calchaquí Norte
}

\author{
María Cecilia PÁEZ \\ Museo de Ciencias Naturales (FCNyM, UNLP), División Arqueología - CONICET \\ ceciliapaez@conicet.gov.ar \\ Marco GiovannetTi \\ Museo de Ciencias Naturales (FCNyM, UNLP), División Arqueología - CONICET \\ mgiovannetti@conicet.gov.ar \\ Rodolfo RAFFINO \\ Museo de Ciencias Naturales (FCNyM, UNLP), División Arqueología - CONICET \\ rraffino@fcnym.unlp.edu.ar
}

Recibido: 2 de marzo de 2011

Aceptado: 18 de abril de 2012

\section{RESUMEN}

Las Pailas constituye uno de los sitios arqueológicos con evidencias de explotación agrícola de mayor extensión y relevancia en los Valles Calchaquíes. La superficie cultivada habría alcanzado cerca de 500 ha, regadas por una compleja red de canales que distribuye el agua proveniente del deshielo de los picos nevados. El área agrícola se divide en dos sectores denominados Las Pailas 1 y 2, separados por un cordón montañoso que es atravesado por un camino arqueológico. Esta vía de comunicación entre ambos sectores encuentra continuidad hacia el oeste hasta llegar al Nevado de Cachi. El reconocimiento y estudio de la configuración agrícola del sitio constituye el punto de partida para analizar la relación de los pobladores con la tierra a lo largo de los dos milenios de ocupación de Las Pailas.

Palabras clave: Valle Calchaquí Norte, tecnología agrícola, campos de cultivo, redes de riego.

\section{Las Pailas. New Contributions to the Understanding of Prehispanic Agriculture in the Northern Calchaqui Valley}

\begin{abstract}
Las Pailas is one of the most extensive and most important archaeological sites with evidence of farming at the Calchaquí Valley. The cultivated area has been of 500 ha, irrigated by a complex network of canals to distribute water from the melting of snow peaks. The agricultural area is divided into two sections called Las Pailas 1 and 2, separated by a mountain range which is crossed by an archaeological way. This channel of communication between the two sectors is continuing west to get to Nevado de Cachi. The survey and study of the agricultural setting of the archaeological site is the starting point for analyzing the relationship of people to land during two millennia of occupation in Las Pailas.
\end{abstract}

Key words: Northern Calchaqui Valley, agricultural technology, farm fields, irrigation network.

Sumario: 1. Introducción. 2. Trabajo de campo y mapeo del sitio. 3. Las Pailas 1. 4. Las Pailas 2. 5. Consideraciones finales. 6. Referencias bibliográficas. 


\section{Introducción}

El valle Calchaquí Norte presenta una de las más notables densidades de restos arqueológicos del Noroeste Argentino (NOA), especialmente en el período Tardío (siglos X a XV) e Inka (parte del siglo XV y primer tercio del XVI). Son más de una veintena sitios que han sido estudiados desde fines del siglo XIX, concentrando en la actualidad gran cantidad de estudios (Ambrosetti 1906, 1907; Boman 1908; Debenedetti 1908; Dillenius 1909; Difrieri 1948; Tarragó y Díaz 1972, 1977; Tarragó y Núñez Regueiro 1972; Tarragó y De Lorenzi 1976; D’Altroy et al. 2000; Williams 2002-2005; Acuto y Gifford 2007).

El estudio de la producción agrícola en el valle Calchaquí Norte ha recibido poca atención a excepción de los primeros trabajos de Tarragó $(1977,1978)$ en la localidad arqueológica de Las Pailas, sitio sobre el que trabajaremos en esta presentación. En cambio, en el resto del NOA el estudio de la agricultura prehispánica ha encontrado nodos de importancia fundamental. En este sentido es reconocida la extensión y características de los sitios Coctaca y Rodero (Albeck y Scattolin 1991; Nielsen 1995) con una superficie de aproximadamente 6.200 ha de cuadros y terrazas de cultivo, lo que les convierte probablemente en los sitios más extensos e importantes de la región en términos de potencial agrícola. En el valle de Cajón (Catamarca) han podido registrarse testimonios de agricultura con sectores de andenes que corresponderían al momento inka en la localidad arqueológica de La Hoyada (de Hoyos 1996). En Catamarca el sitio Los Colorados, cercano y articulado a El Shincal, muestra una amplia extensión de terrenos cultivados utilizando las laderas de los cerros mediante andenería y canchones sobre planicies a la vera del río Quimivil (Giovannetti 2009, Giovannetti et al. 2007). Paralelamente, el estudio del manejo hidráulico en relación al regadío ha alcanzado un buen desarrollo incluso para momentos tempranos de la práctica agrícola. En tiempos Aguada, por ejemplo, se habría desarrollado a través de andenes y terrazas una agricultura que requería estructuras de recolección, almacenamiento y traslado de agua para asegurar eficazmente los cultivos (Kriscautsky 19961997; Puentes 2003). Para el primer milenio d.C. se registraron importantes obras de arquitectura hidráulica en la Puna, tal como aparece documentado en Laguna Blanca (Díaz 2009) y Tebenquiche Chico (Quesada 2006, 2007). A esto hay que agregar los importantes trabajos en Casabindo (Jujuy), donde tanto las faldas de los cerros como los fondos de valle y quebradas en Capinte, Potrero y Tarante fueron aprovechados extensamente para el cultivo con andenes y canchones, con notables obras de riego a través de canales de gran extensión (Albeck 1995a, 1995b). En Salta, próximo a nuestra región de estudio, existen algunos antecedentes para la zona de Molinos donde aprovechando el cauce del río homónimo se habría modificado el paisaje para el cultivo a través del uso de amplios cuadros asociados a períodos pre Tardíos (Baldini y Villamayor 2007).

En este trabajo nos proponemos presentar los resultados de las últimas investigaciones realizadas en el área del yacimiento arqueológico Las Pailas, las cuales se orientan a evaluar el potencial agrícola del sitio a partir de un análisis arquitectónico y espacial de la infraestructura de cultivo. Esto último comprende dos componentes 
fundamentales como son las redes hidráulicas y los campos agrícolas propiamente dichos.

El sitio arqueológico Las Pailas se ubica aproximadamente $16 \mathrm{~km}$ al NO de la población de Cachi (Salta). La descripción realizada en los años 70 se corresponde con el sector mayoritario del sitio compuesto por un poblado semi-conglomerado y una amplia extensión agrícola circundante de aproximadamente 300 ha. Este sector es atravesado por los ríos Potrerillos y Peñas Blancas que nacen a partir de las aguas de deshielo del nevado de Cachi y se transforman luego en el río Las Arcas, tributario del Cachi. Estos cuerpos de agua permanente dan un carácter importante a la zona desde el punto de vista de su riqueza hídrica y es lo que posibilita la mayor parte de los cultivos actuales (Tarragó 1977).

Los estudios precedentes en el área han mostrado que el sector central se compone de estructuras desiguales asociadas, conformando el poblado semi-conglomerado que habría sido asiento de las distintas actividades desarrolladas por quienes habitaban diariamente el sitio. Hacia el oeste se encuentran cuadros de cultivo, asociados a lo que fuera descrito como canchones (Raffino 1975) ${ }^{1}$, con recintos dispersos entre ellos. En el sector norte continúan estructuras análogas, que hacia el noreste se van matizando con recintos habitacionales similares a los observados en el poblado. Las estructuras ubicadas al este también incluyen cuadros de cultivo y habitaciones simples dispersas, aunque la visibilidad en este sector se ve reducida por las acciones derivadas de la ocupación humana actual. Finalmente, hacia el sur continúan los cuadros agrícolas (ibíd.).

Las características del sitio hacen suponer una ocupación continua desde los inicios del primer milenio, donde el componente agrícola habría desempeñado un papel importante en la estructura y funcionamiento de este espacio. La configuración más tardía, habría estado representada por la ocupación del poblado central articulado con el sector de cultivo. Las evidencias actuales hacen suponer que el sitio habría estado en pleno funcionamiento hasta la conquista española y aún en momentos posteriores.

\section{Trabajo de campo y mapeo del sitio}

En una primera instancia de trabajo se realizó un relevamiento cartográfico basado en imágenes satelitales (QuickBird) obtenidas a partir del navegador Google Earth. El área en cuestión presenta imágenes con una resolución suficiente como para permitir detectar los muros divisores de campos de cultivo de diferentes tamaños, así como de los otros tipos de estructuras dispersas en el área agrícola. Esta herramienta nos permitió trazar un mapa del sitio (Figuras 1 y 11), que luego fue contrastado presencialmente en el terreno.

En sucesivas visitas al campo se corroboraron direcciones cardinales, medidas y atributos de muros visualizados de manera dudosa en la imagen satelital, abarcando la totalidad del área agrícola. Asimismo, se realizaron tres transectas de reconoci-

\footnotetext{
1 Raffino denomina canchón o bancal de cultivo a la parcela de tierra delimitada con paredes de tierra o barro, emplazada sobre terrenos de poca pendiente, de 2 a $8 \%$. Se dispone generalmente en forma longitudinal a la pendiente.
} 
miento detallado muestreando cada $100 \mathrm{~m}$ las características arquitectónicas de las estructuras dentro de la misma. Las transectas se realizaron en dirección NO-SE cubriendo un total de $6 \mathrm{~km}$ y abarcando un amplio espectro de estructuras asociadas al cultivo. Las variables consideradas en el muestreo incluyeron: tipo y características métricas de la estructura (ancho y largo); pendiente del sector; características de los muros (tipo, ancho, alto, material, ángulos y técnica constructiva); relación espacial con otras estructuras próximas; presencia/ausencia de estructuras de riego. Los datos fueron volcados en una planilla confeccionada a tal efecto que incluyó, asimismo, datos de coordenadas geográficas basados en tecnología GPS y mapeo a mano alzada de cada unidad. El registro fue complementado con información fotográfica y recolecciones superficiales de material arqueológico dentro de cada unidad y en el trayecto entre unidades.

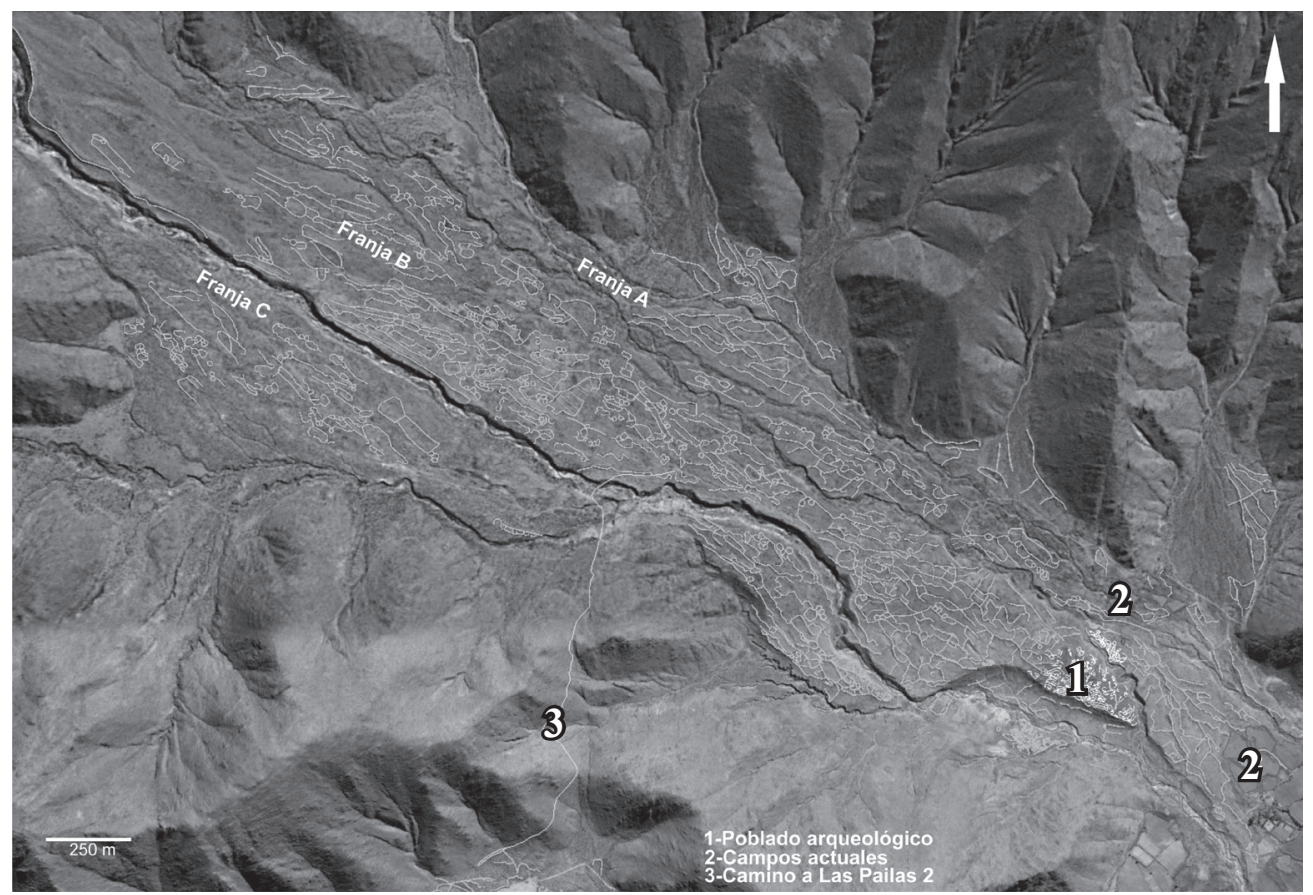

Figura 1: Cartografía de Las Pailas 1 indicando los campos agrícolas arqueológicos y actuales y las estructuras correspondientes al poblado semi-conglomerado.

\section{Las Pailas 1}

\subsection{Campos agrícolas}

Las Pailas 1 representa al sector que fuera descrito por Tarragó $(1976,1977)$ a partir de sucesivos trabajos de campo en la década de 1970. El sitio se localiza en un valle que corre en dirección NO-SE. Según nuestros cálculos, a través de las imágenes satelitales y 
prospecciones en el campo, cubre una superficie de aproximadamente 390 ha, desde los campos cultivados en la actualidad -que probablemente correspondan a estructuras arqueológicas reutilizadas- $\left(25^{\circ} 02^{\prime} 05^{\prime}\right.$ 'S; 66 $\left.6^{\circ} 13^{\prime} 25^{\prime \prime} \mathrm{W}\right)$ hasta las cercanías del Nevado de Cachi ( $25^{\circ} 00^{\prime} 53^{\prime \prime} \mathrm{S}, 66^{\circ} 15^{\prime} 39^{\prime}$ 'W). Las diferencias de altitud entre ambos extremos van desde $2.960 \mathrm{msnm}$ hasta $3.380 \mathrm{msnm}$, correspondiendo este punto al último sector con estructuras agrícolas divisado en el extremo NO cerca del pie del nevado. Hacia el E y O está bordeado por dos líneas de serranías que confluyen con las estribaciones del Nevado de Cachi (ver Figura 1).

Las actuales ruinas arqueológicas ven alterada su fisonomía en algunos sectores debido a la reutilización de superficies de cultivo y canales de riego, además de algunas sendas para el pastoreo de animales y vías de comunicación. Hacia el SE del sitio el paisaje de fincas e instalaciones residenciales muy probablemente se corresponda con la configuración agrícola prehispánica, sugiriendo la posibilidad de que el sitio haya abarcado una extensión mucho mayor que la observada en la actualidad.

Para el estudio de los campos de cultivo, en la búsqueda de un mejor ordenamiento espacial, se dividió el sitio en tres franjas paralelas. A partir de los ríos Potrerillos y Peñas Blancas es posible seccionar el espacio al costado y NO del poblado en tres franjas A, B y C (ver Figura 1) que, aunque de diferente tamaño, permiten una mejor descripción de las áreas.

Los campos agrícolas reconocidos mostraron diferente morfología, características y dimensiones a lo largo de la superficie prospectada aunque fue posible percibir algunos patrones generales y distinguirlos según zonas específicas. En general en los sectores más próximos al poblado semi-conglomerado hasta aproximadamente $800 \mathrm{~m}$ en dirección NO las unidades presentan menores dimensiones, son subcuadrangulares -con ángulos redondeados- (Figuras 2 y 3), o en algunos casos irregulares (Figura 4), se encuentran próximas entre sí sin pérdida de continuidad, con técnicas constructivas que dan cuenta de mayor inversión de tiempo y trabajo. Más allá de esta distancia los recintos de cultivo se presentan, en líneas generales, alargados con morfologías rectangulares a subrectangulares en algunos casos y circulares grandes en otros. Se observa un marcado predominio del largo sobre el ancho, coincidiendo el primero con la pendiente (Figura 5). Es remarcable que en todo el sitio hay desniveles entre un cuadro y otro, buscando en cada caso el máximo de horizontalidad como medida para favorecer los cultivos y disminuir la erosión, a lo que se agrega una correspondencia entre las paredes de las estructuras y las curvas de nivel (Tarragó 1977). Por otro lado en la mayoría de los canchones existen aperturas para el paso de agua de riego. Se trata de cortes sobre la continuidad de los muros de pirca (Figura 2), presentándose por lo general sobre los muros más ordenados ya sean de tipo doble o simple y no así en los amontonamientos de despedrado.

En la subzona más distante del poblado hay, además, mayor cantidad de espacios abiertos y los campos presentan una densidad menor de muros de despedrado, observándose inclusive en algunos sectores superficies cubiertas de pequeños cantos rodados. Esta característica distingue este sector de la primer subzona donde la ausencia de cantos rodados en el interior de los campos es notable.

Si bien en la primer subzona se distinguieron recintos circulares pequeños al interior y en los laterales de los canchones de cultivo (Figura 2), la concentración de los mismos en la segunda subzona se amplifica significativamente (Figura 5). Aquí se encuentran unidos 
tanto individualmente a los cuadros como dispuestos en conjuntos de 2 a 6 unidades. La mayor parte de estos recintos tienen medidas que oscilan aproximadamente entre $10 \mathrm{~m}$ y $20 \mathrm{~m}$, registrándose variaciones por encima y por debajo del rango referido. Las características arquitectónicas así como su vinculación espacial con los cuadros sugieren que, al menos en algunos casos, podrían estar relacionados al almacenamiento.

Otra de las diferencias registrada entre ambas subzonas es la frecuencia en el registro de artefactos, tanto cerámicos como líticos, presentando una disminución perceptible correlativa con el alejamiento del poblado semi-conglomerado. La cerámica fue asignada a estilos de momento temprano y tardío, además de algunos fragmentos que podrían corresponder a los estilos incaicos regionales. La abundancia de palas y azadas líticas -además de lascas e instrumentos tallados- se corresponde con el tipo de actividades que se presume para el sector analizado ${ }^{2}$.

El tipo de técnica constructiva empleada a lo largo del sitio combina muros simples (apilamiento de cantos rodados en una sola hilera), dobles (apilamiento doble de cantos rodados con mortero de barro y piedras pequeñas), y/o amplias extensiones a manera de muros, pero irregulares, producto de la acumulación por prácticas de despedrado. Este último tipo de estructuras, que ya fueran descritas por Tarragó (1977), pueden alcanzar hasta $8 \mathrm{~m}$ de ancho, encontrando continuidad entre un canchón y otro. Es común que los cuadros concentren los tres tipos de paredes divisorias, aunque el uso del muro simple es habitual para las divisiones internas de las estructuras, o los aterrazamientos que cubren parcialmente algunas de ellas.

De esta manera pudieron determinarse 8 tipos de unidades basándose en la presencia y asociaciones de las técnicas constructivas empleadas:

1. Asociación de muro simple, doble y despedrado en la conformación de los canchones, con unidades internas como aterrazamientos, estructuras circulares o subcirculares (Figura 2).

3. Asociación de muro doble y despedrado en las paredes de los canchones, con unidades internas de muro simple.

4. Asociación de muro doble y despedrado en las paredes de los canchones, sin unidades internas.

5. Asociación de muro simple y despedrado en las paredes de las estructuras, con unidades internas de muro simple (Figura 4).

6. Asociación de muro simple y despedrado en las paredes de las estructuras, sin unidades internas.

7. Estructuras conformadas exclusivamente por muros de despedrado, con estructuras internas de muro simple (Figura 5).

8. Estructuras conformadas exclusivamente por muros de despedrado, sin estructuras internas.

\footnotetext{
${ }^{2}$ Los materiales mencionados están siendo actualmente analizados, por lo que la información que aquí se ofrece es limitada.
} 


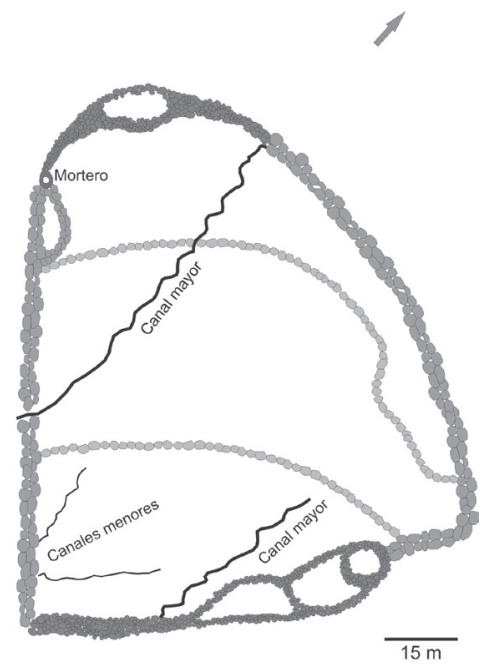

Muro simple

Muro doble

Muro de despedrado

Muro simple

Muro doble

Muro de despedrado

Figura 2: Canchón de cultivo correspondiente a la unidad de muestreo 4, combinando tres tipos de muro (simple, doble y despedrado) con unidades subcirculares asociadas.

Figura 3: Canchón de cultivo correspondiente a la unidad de muestreo 6 , combinando tres tipos de muro (simple, doble y despedrado).

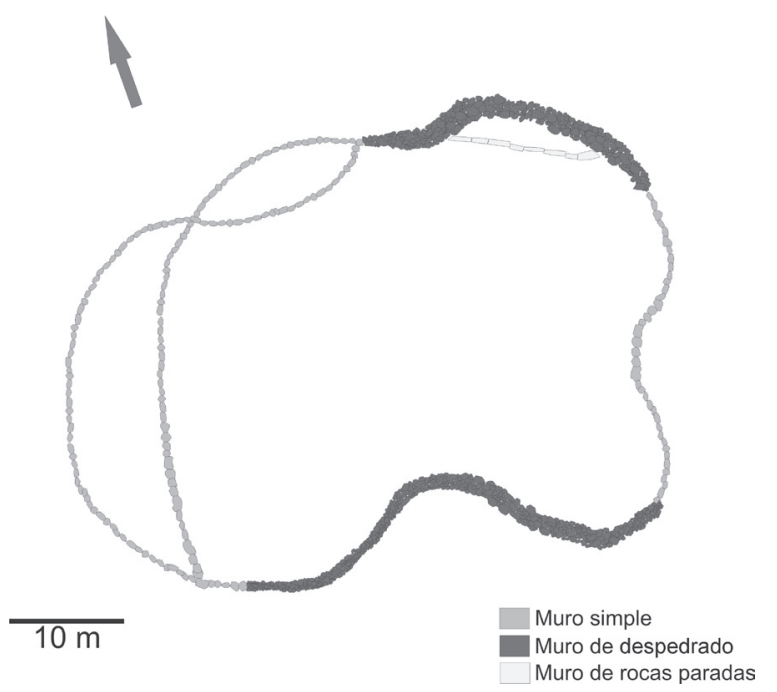

Figura 4: Canchón de cultivo correspondiente a la unidad de muestreo 10, combinando muro simple y despedrado, con unidades en su interior. 


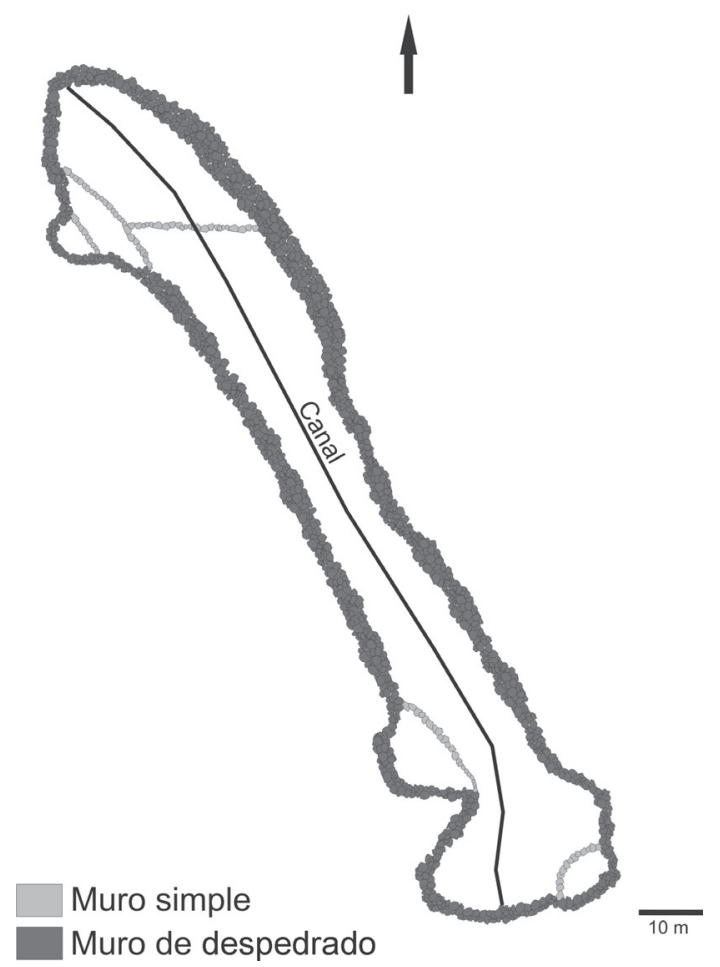

Figura 5: Canchón de cultivo alargado correspondiente a la unidad de muestreo 11, conformado por muros de despedrado y subdivisiones internas de muro simple.

La presencia de instrumentos de molienda es una característica recurrente en todo el sitio, que se hace notoria en las estructuras que componen el poblado arqueológico. En el sitio se pudieron individualizar tanto morteros ${ }^{3}$ como conanas $^{4}$, en tanto los primeros están destinados a la obtención de granos triturados, las segundas están relacionadas con el procesamiento de los elementos a estado de harinas (Babot 2004; Giovannetti 2009; Nardi y Chertudi 1969, 1970). La frecuencia aparición de conanas en el poblado es notablemente mayor que la de morteros, estos últimos compuestos generalmente por uno, dos y tres orificios, tanto en soportes móviles como fijos.

Para el caso del sector agrícola se invierte la relación, correspondiendo la mayor parte de las estructuras a morteros, ubicados en el interior de los cuadros o próximos a los recintos circulares. Contienen una o dos oquedades dispuestas sobre rocas (Figura 6), mayormente fijas. Espacialmente se disponen en los cuadros más cercanos al poblado, lo que sugiere que además de las prácticas de cultivo propiamente dichas, en estos espacios se estaba llevando a cabo al menos alguna de las etapas de procesamiento de los granos dentro de la actividad productiva.

3 Oquedades circulares cupuliformes para el tratamiento de los productos por machacamiento.

4 Oquedades elongadas para el tratamiento del producto por abrasión. 


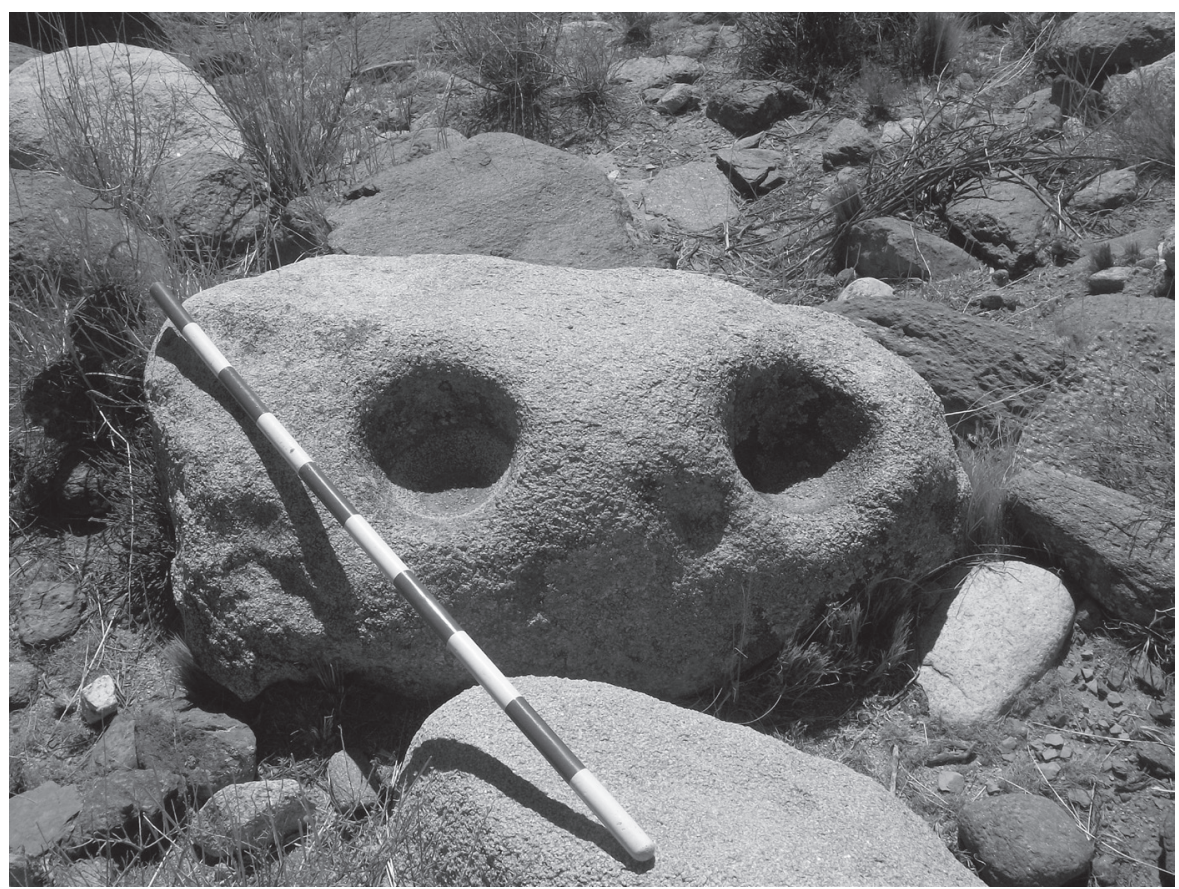

Figura 6: Mortero múltiple con unidades cupuliformes, ubicado en el interior de los campos agrícolas.

\subsection{La red hidráulica en Las Pailas 1}

En las redes de riego clásicas, es decir, aquellas que en términos hidrológicos el agua es transportada sólo por gravedad, existen varios aspectos para describir y clasificar las partes componentes, claro está en términos modernos. Luego de la toma de agua (el primer componente de esta red) es el canal principal el que conducirá el flujo hasta los canales secundarios que abastecerán el espacio entre dos vaguadas a través de canales terciarios. Los canales de último orden, las acequias de distribución o regueras, conducen el agua directamente a las parcelas de cultivo. Finalmente un canal de desagüe retornaría el agua no utilizada al mismo río desde donde se extrajo (Poiree y Ollier 1974).

Pueden encontrarse los mismos componentes con otras denominaciones, también modernas, pero que han sido utilizadas para descripciones arqueológicas (Albeck 1995a, 1995b; Damiani 2002; Denevan 1980). Canales matrices de primer orden corresponderían a los primarios y se caracterizarían por transportar grandes flujos de agua y finalizarían en un artificio hidráulico que disminuiría la velocidad al entrar en los canales matrices de segundo orden. Estos últimos, y conjuntamente con las hijuelas de riego (canales de tercer orden), transportarían agua a velocidades medias o bajas. Los canales de primer orden suelen conducirse sobre sectores no aptos para la agricultura, muchas veces laderas de los cerros o sectores más altos que los cam- 
pos que se irrigarán. Los restantes conductos suelen atravesar los mismos campos de cultivo hasta morir en las parcelas.

La distribución del agua a través de la superficie de cultivo es una preocupación latente en la actualidad en Las Pailas, cuyo encauzamiento ha requerido el uso de la red prehispánica en algunos tramos del riego de los campos por parte de las familias que viven en las inmediaciones del sitio.

El reconocimiento realizado sobre una parte de los canales arqueológicos dio cuenta de una extensa red hidráulica que distribuye el agua proveniente del deshielo de los picos nevados. Los dos ríos que actualmente recorren el sitio -Potrerillos y Peñas Blancas- obtienen de esta manera su cauce permanente. La mayor parte de los conductos transcurren aéreamente a través de canales principales y secundarios. No obstante, en algunos tramos se pudieron identificar estructuras cuadrangulares (Figura 7) que sugieren que debieron transcurrir de manera subterránea.

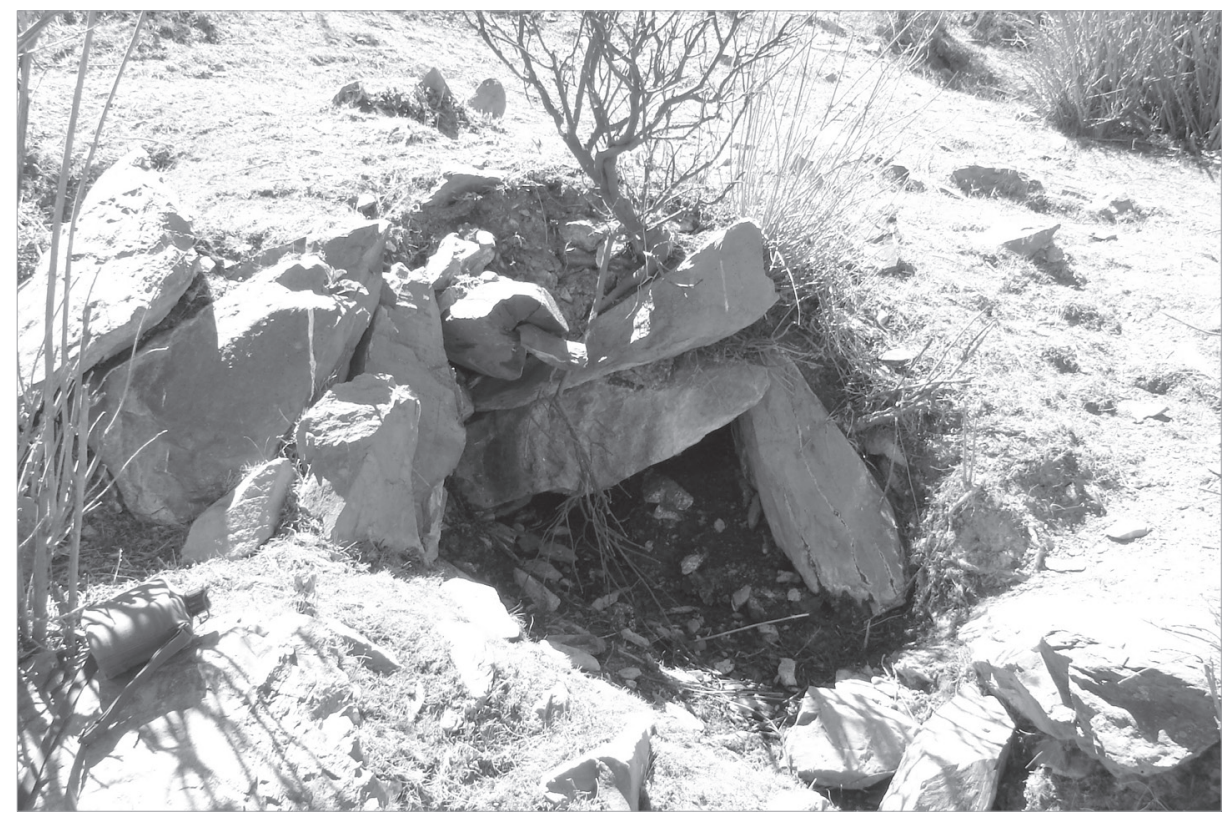

Figura 7: Salida de flujo de agua de canal subterráneo, localizado en Las Pailas 1.

En la Figura 8 es posible distinguir el recorrido de varios de los canales de distinto orden que se detectaron en el campo. En primer lugar, el punto 1 distingue una de las tomas de agua identificadas por nosotros siendo de tipo rústica según la tipología de Poiree y Ollier (1974), es decir, como una mera apertura lateral sobre el cauce del río. Es posible que existan otras a juzgar por la distribución de canales secundarios de acuerdo al esquema de redes mapeado. La toma de agua mencionada se ubica 6,1 $\mathrm{km}$ en dirección NO del poblado arqueológico y se trata de un mero acomodamiento de rocas, cantos rodados de gran tamaño, para permitir el desvío de agua. 


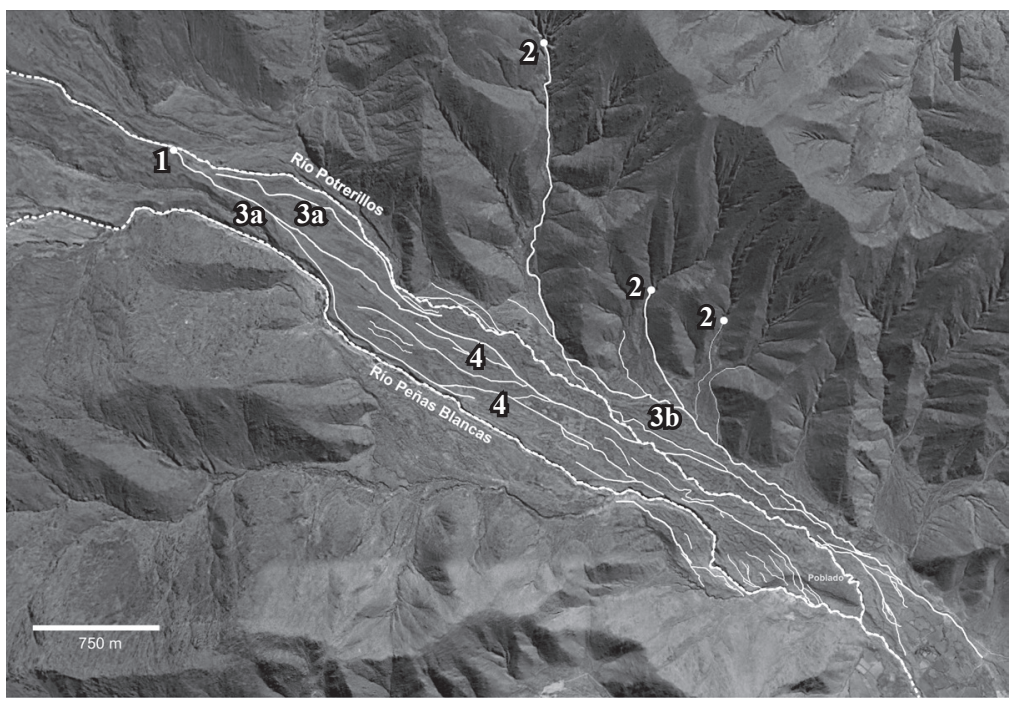

Figura 8: Cartografía de la red hidráulica de Las Pailas 1: (1) toma de agua; (2) conductos de altura tallados en roca base para encauzar el agua; (3a) canal principal originado en el río Potrerillos; (3b) canal principal proveniente de los cursos de altura; (4) canal secundario.

También en relación a la captura de flujo para regadío se ha podido detectar otro interesante sistema que difiere del anterior. En varios cauces de montaña fue posible constatar en las partes altas importantes trabajos de corte y formatización de roca de base (señalados como punto 2 en la Figura 8 ) para lograr un eficaz pasaje del agua (Figura 9). Son conductos de entre uno y dos metros de ancho cuya función es canalizar el agua de lluvia y dirigirla con precisión a los campos de cultivo. Tres de estos sistemas fueron registrados en la ladera NE, pero existe la sospecha de que en cada cauce de montaña exista un sistema similar al menos en el trayecto que contiene los campos de cultivo.

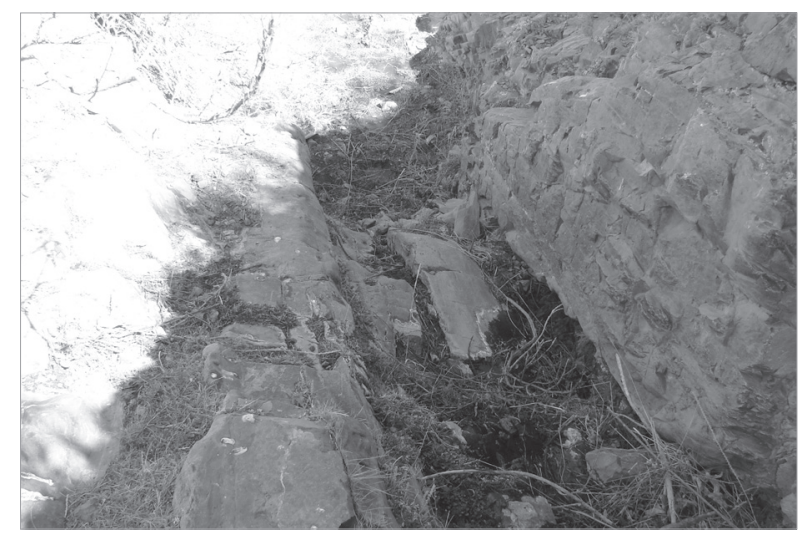

Figura 9: Roca de basamento cortada para el encauzamiento del agua. 
Los canales primarios pueden ser recorridos en su totalidad, es decir desde que parten de la toma de agua en al menos dos casos (puntos 3a en la Figura 8). Uno de ellos se correspondería con la toma de agua mencionada previamente. Es necesario destacar que estos canales sólo abastecen la franja B de los campos de cultivo, es decir la que queda entre los dos ríos del valle y conforma la mayor extensión de tierras con evidencia de agricultura. El agua es tomada solamente del río Potrerillos dado que el río Peñas Blancas presenta sobre su margen izquierda altos paredones que hacen muy difícil construir tomas de agua. Estos canales primarios que mencionamos se componen de aperturas sobre la tierra de aproximadamente seis metros de ancho y profundidades variables de entre uno y dos metros.

El otro tipo de canal primario corresponde a aquel que colecta el agua de las cumbres al NE (punto $3 \mathrm{~b}$ de la Figura 8). Posiblemente la erosión continua de siglos ha producido lo que hoy parece ser el cauce seco de un arroyo temporal que, por momentos, llega a medir $20 \mathrm{~m}$ de ancho, aunque lo normal es que no supere los $12 \mathrm{~m}$. El agua colectada por medio de este sistema pareciera regar principalmente los campos aguas abajo hacia el SE, pasando el poblado y cercanos a los cuadros de cultivo actuales. Se detectan pocos canales que se desprendan de éste y rieguen parte de la superficie de la franja A de Las Pailas 1. Es posible que este canal principal sirviera fundamentalmente para transportar agua a campos de cultivo arqueológicos que hoy se encuentran superpuestos por campos actuales.

Los canales secundarios se desprenden en abundancia de los canales que mencionáramos previamente. A modo de ejemplo hemos marcado sólo algunos en la Figura 8 (puntos 4). En varios casos vemos que los canales secundarios atraviesan transversalmente la franja B para terminar desaguando en otro canal principal. En el trayecto desprenden varios canales terciarios o regueras que podrán morir dentro de un canchón o conectarse con varios canchones y finalmente volcar su flujo en algún conducto de desagüe que llevará el agua nuevamente hacia el río Potrerillos. Los canales secundarios por lo general miden entre 0,40 y $0,60 \mathrm{~m}$, cavados en la tierra de geometría en sección transversal semicircular; pueden llegar hasta $0,50 \mathrm{~m}$ de profundidad.

Al ingresar dentro de los cuadros de cultivo los canales se ramifican en varias regueras o hijuelas de riego, intentando abarcar la mayor superficie posible. En la Figura 10 puede verse este sistema con claridad en uno de los canchones arqueológicos. Estos canales finales miden entre 0,20 y $0,40 \mathrm{~m}$ de ancho y son de escasa profundidad. Finalmente, se detectaron varios canales de desagüe en el sector final de la franja B, ya muy cerca del poblado arqueológico, sobre un costado del río Peñas Blancas donde éste realiza una inflexión hacia el NE. Los canales caen abruptamente por los paredones del margen izquierdo que aún mantienen una altura importante. Algunos de estos canales aún regarán unos pocos canchones localizados en la terraza de inundación antes de confluir definitivamente en el río.

El sistema de transporte de agua más distintivo descubierto en Las Pailas 1 es aquel que transporta agua subterráneamente a través de unos complejos canales totalmente tapizados en roca tanto en paredes como en base y techo. Se pueden visualizar en un caso en un corte del terreno producido por una cárcava y en una salida preparada para evacuar el agua hacia la superficie (ver Figura 7). Desconocemos hasta el momento si las funciones de este dispositivo son las mismas que los canales a cielo abierto. 


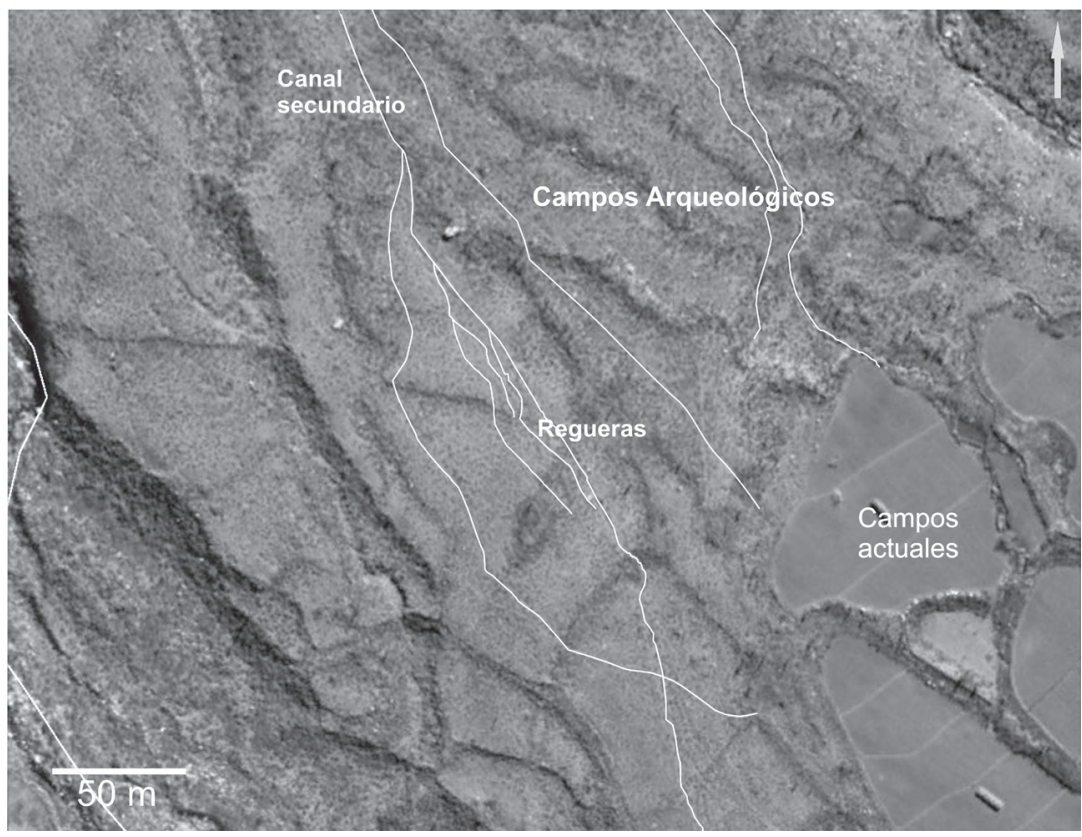

Figura 10: Canales secundarios y regueras que atraviesan los campos arqueológicos.

\section{Las Pailas 2}

Hacia el SO de Las Pailas 1, se levanta un cordón montañoso que se eleva entre $200 \mathrm{~m}$ y $500 \mathrm{~m}$ por encima del sitio. Del otro lado se hace presente un pequeño valle atravesado por un curso de agua que corre en sentido NO-SE, naciendo de las estribaciones meridionales del Nevado de Cachi y siendo captado por el río Las Arcas. Sobre este vallecito se despliega lo que denominamos Las Pailas 2, un sitio similar y conectado al primero por un sendero que atraviesa un paso montañoso. Es usada aún en la actualidad para comunicar ambos sectores, extendiéndose unos 1470 m manteniendo una dirección N-S. La senda se interna entre los canchones para luego dirigirse hacia el oeste directo al nevado de Cachi.

Los campos agrícolas de Las Pailas 2 fueron detectados a partir de las fotografías satelitales y de prospecciones de campo (Figura 11). No obstante, la morfología de los cuadros agrícolas y las características y extensión de la red de riego nos permiten establecer de manera fehaciente una homología directa entre ambos sectores, que debieron funcionar contemporáneamente en algún momento del desarrollo productivo de la región. Pudimos realizar un calculo confiable de aproximadamente 110 ha para este sector.

En relación a la especificidad de los campos agrícolas vemos que presentan cuadros de diferentes morfologías -cuadrangulares, subcuadrangulares e irregulares-, además de estructuras circulares y subcirculares asociadas a los primeros o unidas entre sí en conjuntos de dos o más. 
Amplios muros de despedrado se articulan con paredes simples y/o dobles para conformar las estructuras que se disponen en el angosto valle, de aproximadamente $300 \mathrm{~m}$ de ancho. La superficie cultivada arqueológica encuentra continuidad con los cuadros actuales hacia la desembocadura del arroyo, lo que permite suponer que, como en el caso de Las Pailas 1, ésta podría ser mayor que lo estimado en base a estructuras arqueológicas.

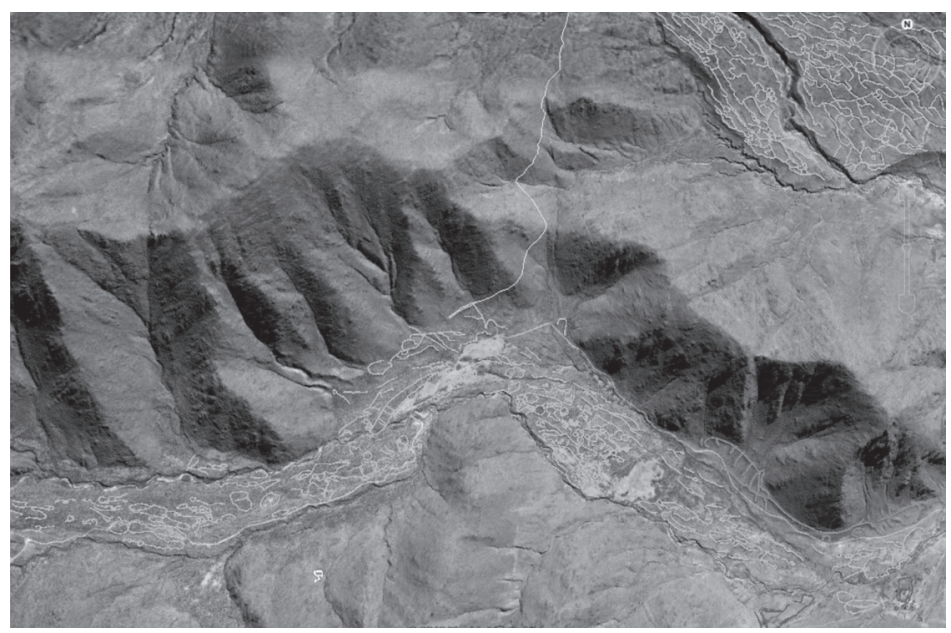

Figura 11: Cartografía de Las Pailas 2 indicando los campos agrícolas arqueológicos y actuales y el camino que conecta con Las Pailas 1.

No pudimos individualizar en un primer recorrido por el campo, estructuras similares a las correspondientes al poblado semiconglomerado mencionado previamente en este trabajo. Sólo pudo individualizarse un sector que presentaba recintos de menores dimensiones y mayor aglutinamiento que el resto de los estructuras, aunque sus características no permitirían asociarlo a una función residencial.

La red de canales parece presentar menor complejidad que Las Pailas 1 debido posiblemente a la cercanía inmediata del arroyo a los campos de cultivo, lo que permitió establecer múltiples tomas con canales de recorridos cortos. Además, como mencionábamos arriba, el ancho del valle es relativamente angosto.

Prácticamente no se registraron elementos de molienda, hecho que era importante en los campos del otro lado de la serranía. De forma análoga, la frecuencia en la superficie de fragmentos cerámicos es menor que en Las Pailas 1 y, en su mayoría, correspondientes a estilos de momentos tardíos.

\section{Consideraciones finales}

A partir de la información presentada a lo largo del trabajo, nos interesaría resaltar algunos aspectos que consideramos sustanciales para abordar la problemática agrícola 
de este sector del Valle Calchaquí. Debemos tener en cuenta que las investigaciones en las que se enmarca este trabajo están en sus inicios, por lo que algunos aspectos con seguridad se irán profundizando en el devenir de los estudios y análisis pormenorizados.

En primer término es remarcable la amplia extensión cultivada del sitio -incluyendo los sectores Las Pailas 1 y 2-, cuyos cálculos de área suman en total cerca de 500 ha. Este valor coloca a Las Pailas entre los sitios de mayor envergadura agrícola en el Noroeste Argentino. Esto pudo ser estimado gracias a realización de la cartografía detallada de toda la localidad arqueológica aquí presentada. En este sentido, los avances tecnológicos de fotografías aéreas y satelitales están realizando un aporte indispensable para el reconocimiento preciso de grandes áreas, como el caso de Las Pailas, cuyo mapeo en el terreno demandaría de excesivo tiempo y esfuerzo.

La incorporación de un nuevo sector (Las Pailas 2) a las investigaciones que se realizaran en la década de los años 70 sobre lo que ahora consideramos Las Pailas 1, constituye otro significativo aporte. En este sentido, este nuevo espacio ya habría sido detectado a partir de fotografías aéreas en aquel momento (Tarragó comunicación personal 2010) aunque no fuera prospectado. Aquí proponemos que habría estado plenamente articulado con Las Pailas 1 dado que no encontramos diferencia sustancial entre los sectores, sobre todo desde el punto de vista de la arquitectura agrícola. La amplitud y características de la superficie cultivada nos permiten hipotetizar la necesidad, en algún momento de la ocupación prehispánica del sitio, de un máximo aprovechamiento de las superficies aptas para la agricultura, generada a partir de la existencia de cursos de agua permanente. Es por ello que se decide transformar el paisaje de angostos valles, tapizándolos completamente de campos agrícolas.

Otro aspecto a destacar es la variabilidad morfométrica y constructiva de las unidades agrícolas muchas veces traducidas en canchones simples y otros con unidades de diferente clase adosadas sobre los laterales o incorporadas en su interior. Dentro de estas últimas destacan estructuras circulares o subcirculares a veces aisladas y otras veces en conjuntos a manera de ramilletes. Desconocemos por el momento su función específica pero hipotetizamos que algunas de ellas pudieran vincularse al almacenaje de los productos cultivados ${ }^{5}$.

El cultivo dentro de los campos habría requerido, además de la horizontalización de la superficie de suelo productivo (Tarragó 1977), la creación de diversos pisos o aterrazamientos en el interior de los canchones a través de los cuales transcurriría el agua de riego por canales cavados en la tierra. En algunos casos, estas terrazas estuvieron directamente asociadas a estructuras de molienda en rocas fijas, dando cuenta de que al menos una parte de lo producido era también procesado en los campos. En este sentido, no pueden dejar de mencionarse las diferencias que atañen a la desigual distribución de los morteros y conanas a lo largo de la superficie agrícola en Las Pailas 1. La mayor frecuencia registrada en sectores aledaños al poblado, y la ausencia en los campos más alejados, hacia el límite NO, hacen suponer que las zonas más próximas al poblado eran objeto de múltiples actividades más allá del cultivo en sí mismo. Incluso, es importante mencionar que la diferencia que se percibió entre

5 Una de ellas ha sido objeto de un sondeo prospectivo y los resultados se encuentran en procesamiento. 
conanas y morteros, siendo estos últimos más comunes en los campos, alienta interpretaciones de que habrían sido más frecuentes algunas etapas de procesamiento de cultivos en los campos, como el machacado o la fractura de granos. En cambio, las etapas de producción de harinas habrían sido habituales en el poblado.

Hemos mencionado en varias oportunidades la reutilización de los campos por parte de los actuales pobladores del área, sobre todo hacia el sector sur ya sobre el río Las Arcas. En la mayor parte de los casos se han reutilizado tanto los campos como los canales. Estos últimos han sido por tramos reacondicionados con cemento, aunque las características y dinámica respetan la tecnología prehispánica. Sobre las imágenes satelitales ha sido posible detectar con claridad que, en medio de los campos actuales, persisten estructuras prehispánicas que no han sido modificadas. Esto da sustento a la propuesta de que la dimensión de los campos agrícolas arqueológicos de Las Pailas habría sido considerablemente mayor, extendiéndose a lo largo del río Las Arcas. Las investigaciones actuales deberán tener en cuenta estos antiguos vestigios, pequeños y aislados, pero valiosos en el momento de dimensionar el alcance de la maquinaria productiva como parte de la estructura de sociedades y paisajes a lo largo de dos milenios en el Valle Calchaquí Norte.

Agradecimientos: Este trabajo ha sido posible gracias al financiamiento del Consejo Nacional de Investigaciones Científicas y Técnicas y al apoyo de la Dirección de Antropología de la Provincia de Salta y el Museo Arqueológico Pío Pablo Díaz. Un especial agradecimiento concierne a la Dra. Myriam Tarragó por sus comentarios e información compartida en relación al sitio. También a los pobladores de Las Pailas, en especial a Milagros Liquín. La responsabilidad de lo expresado es exclusiva de los autores.

\section{Referencias bibliográficas}

Acuto, Félix A. y Chad Gifford

2007 «Lugar, arquitectura y narrativas de poder: relaciones sociales y experiencias en los centros Inkas del Valle Calchaquí norte». Arqueología Suramericana 3 (2): 135-161.

Albeck, María Ester

1995a «Tecnología Agrícola e Hidráulica en Casabindo, Prov. de Jujuy, Argentina». Hombre y Desierto. Una Perspectiva Cultural 9 (1): 257-268.

1995 b «Funcionalidad y cronología relativa de los recintos de cultivo de Coctaca, Prov. de Jujuy, Rca. Argentina». Hombre y Desierto. Una Perspectiva Cultural 9 (1): 317-323.

Albeck, María Ester y María Cristina Scattolín

1991 «Cálculo fotogramétrico de superficies de cultivo en Coctaca y Rodero, Quebrada de Humahuaca». Avances en Arqueología 1: 43-58.

AmbrosetTi, Juan Bautista

1906 Exploraciones arqueológicas en Pampa Grande (Provincia de Salta). Publicación de la Sección Antropológica 1. Buenos Aires: Facultad de Filosofía y Letras.

1907 Exploraciones arqueológicas en la ciudad prehistórica de «La Paya» (Valle Calchaquí, Provincia de Salta). Publicación de la Sección Antropológica 3. Buenos Aires: Facultad de Filosofía y Letras. 
ВАвот, María del Pilar

2004 Tecnología y utilización de artefactos de molienda en el Noroeste Prehispánico. Tesis doctoral inédita. Instituto de Arqueología y Museo. Tucumán: Universidad Nacional de Tucumán.

BALDINI, Lidia y Virginia VILLAMAYOR

2007 «Espacios productivos en la cuenca del río Molinos (Valle Calchaquí, Salta)». Cuadernos de la Facultad de Humanidades y Ciencias Sociales 32: 35-51.

BOMAN, Eric

1908 Antigüedades de la región andina de la República Argentina y del desierto de Atacama. San Salvador: Universidad Nacional de Jujuy.

D’Altroy, Terence, Ana María Lorandi, Verónica Williams, Milena Calderari, Christine A. Hastorf, Elizabeth DeMarrais y Melissa Hagstrum

2000 «Inka Rule in the Northern Calchaqui Valley, Argentina». Journal of Field Archaeology 27 (1): 1-26.

DAMIANi, Oscar

2002 «Sistemas de riego prehispánico en el valle de Iglesia, San Juan, Argentina». Multequina, Latin American Journal of Natural Resources 11: 1-38.

De Hoyos, María

1996 «La Hoyada. Un enclave de producción agrícola en el valle del Cajón, provincia de Catamarca». Revista del Museo de Historia Nacional de San Rafael 25 (3-4): 273-293.

DEBENEDETTI, Salvador

1908 Excursión arqueológica a las ruinas de Kipón (Valle Calchaquí, Provincia de Salta). Publicaciones de la Sección Antropológica 4. Buenos Aires: Facultad de Filosofía y Letras.

DenEvan, William

1980 «Tipología de configuraciones agrícolas prehispánicas». América Indígena 40 (4): 619-652.

DíAz, Raúl Alejandro

2009 Historias de Agua y Tierra: Introducción a los espacios agrícolas prehispánicos de Laguna Blanca. El caso de estudio de la Aldea Arqueológica Piedra Negra (Departamento Belén-Provincia de Catamarca). Tesis de Licenciatura inédita, Escuela de Arqueología. San Fernando del Valle de Catamarca: Universidad Nacional de Catamarca.

DIFRIERI, Horacio A.

1948 «Las ruinas de Potrero de Payogasta (Provincia de Salta, Argentina)», Actes du XXVIII Congrès International des Américanistes, pp. 599-603. París.

Dillenius, Juliane A.

1909 Observaciones arqueológicas sobre alfarería funeraria de la Poma (valle Calchaquí, Provincia de Salta). Publicaciones de la Sección Antropológica 5. Buenos Aires: Facultad de Filosofía y Letras.

Giovannetti, Marco Antonio

2009 Articulación entre el sistema agrícola, redes de irrigación y áreas de molienda como medida del grado de ocupación Inka en El Shincal y Los Colorados (prov. 
de Catamarca). Tesis doctoral inédita. Facultad de Ciencias Naturales y Museo. La Plata: Universidad Nacional de La Plata.

Giovannetti, Marco Antonio, Reinaldo Moralejo y Gustavo Corrado

2007 «Informe preliminar y presentación del sitio Los Colorados (Depto. Belén, Catamarca)», en Memoria del III Congreso de Historia de Catamarca, Tomo I, pp. 125-150. San Fernando del Valle de Catamarca.

KRISCAUTSKY, Nestor

1996-97 «Sistemas productivos y estructuras arqueológicas relacionadas con la producción agropecuaria en el Valle de Catamarca». Shincal 6: 65-69.

NARDI, Ricardo y Susana Chertudi

1969 «Instrumentos arcaicos para majar y moler en San Juan (Argentina)». Revista de Etnografía 26: 387-418.

1970 «Instrumentos arcaicos para majar y moler en San Juan (Argentina)». Revista de Etnografía 27:137-188.

Nielsen, Axel E.

1995 «Aportes al estudio de la producción agrícola Inka en la quebrada de Humahuaca (Jujuy, Argentina)». Hombre y Desierto. Una perspectiva cultural 9 (1): 245-260.

PoIrée, Maurice y Charles Ollier

1974 El regadio. Redes, teoría, técnica y economía de los riegos. Barcelona: Editores Técnicos Asociados S. A.

Puentes, Hugo

2003 Los primeros tiempos del formativo en el valle de Catamarca. Control de cuenca, manejo hidráulico y uso del espacio. Un caso de estudio: sitio El Tala (Dpto. Capital, Catamarca). Tesis doctoral inédita. Escuela de Arqueología. San Fernando del Valle de Catamarca: Universidad Nacional de Catamarca.

Quesada, Marcos N.

2006 «El diseño de las redes de riego y las escalas sociales de la producción agrícola en el 1er milenio DC (Tebenquiche Chico, Puna de Atacama)». Estudios Atacameños 31: 31-46.

2007 Paisajes agrarios en el área de Antofalla. Procesos de trabajo y escalas sociales de la producción agrícola (I y II Milenio d.C.). Tesis doctoral inédita. Facultad de Ciencias Naturales y Museo. La Plata: Universidad Nacional de La Plata.

RAFFino, Rodolfo A.

1975 «Potencial ecológico y modelos económicos en el Noroeste argentino». Relaciones 9: 21-43.

TARRAGó, Myriam N.

1977 «La localidad arqueológica de Las Pailas, provincia de Salta, Argentina», en Actas del VII Congreso de Arqueología de Chile Vol. II, pp. 499-517. Altos de Vilches.

1978 «Paleoecology of the Calchaquí Valley, Salta Province, Argentina», en Advances in Andean Archaeology, D. Browman ed., pp. 485-512. La Haya: Mouton Publishers.

TARragó, Myriam N. y Mónica De Lorenzi

1976 Arqueología del Valle Calchaquí. Etnia 23-24: 1-35. 
TARragó, Myriam N. y Pío Pablo Díaz

1972 «Sitios arqueológicos del Valle Calchaquí». Estudios de Arqueología 1: 49-61.

1977 «Sitios arqueológicos del Valle Calchaquí». Estudios de Arqueología 2: 63-71.

TARRAGó, Myriam N. y Víctor A. NúÑEz REgueiro

1972 «Un diseño de investigación arqueológica sobre el Valle Calchaquí: Fase exploratoria». Estudios de Arqueología 1: 62-85.

WiLLiams, Verónica.

2002-05 «Provincias y Capitales. Una visita a Tolombón, Salta, Argentina». Xama 15, 16, 17, 18: 177-198. 